\title{
Scalable Reading
}

\author{
Thomas Weitin
}

Online publiziert: 8. März 2017

(C) J.B. Metzler, Part of Springer Nature, Springer-Verlag GmbH 2017

Anfang Juni 2015 fand in der Seeburg am Schweizer Bodenseeufer eine Arbeitstagung statt unter dem Titel: »Scalable Reading. Paul Heyses Deutscher Novellenschatz zwischen Einzeltext und Makroanalyse«. Dazu waren den Teilnehmerinnen und Teilnehmern im Vorfeld nicht nur alle 86 Novellen der historischen Sammlung übersandt worden, die Heyse gemeinsam mit Hermann Kurz zwischen 1871 und 1876 herausgegeben hat. Bereitgestellt wurde die Sammlung auch als ein nach dem Standard der Text Encoding Initiative (TEI) annotiertes xml-Korpus sowie im einfachen txt-Format. Die doppelte Vorbereitung sollte es ermöglichen, hermeneutisch und quantitativ arbeitende Kolleginnen und Kollegen über den gemeinsamen historischen Gegenstand miteinander ins Gespräch zu bringen. Die äußerst lebhaften Debatten auf der Tagung ${ }^{1}$ haben gezeigt, wie notwendig und lohnenswert derart fundierte Fachgespräche für die Literatur- und Sprachwissenschaft sind, weil sie es erlauben, die aktuellen methodischen Kernprobleme unserer Fächer genau in den Blick zu nehmen.

Die Frage, wie sich die Einzeltextlektüre mit der Analyse größerer und großer Textmengen gewinnbringend kombinieren lässt, wird in den kommenden zehn Jahren nicht nur in den Geisteswissenschaften zentral sein. Sie lässt sich nicht theoretisch klären, verlangt aber viel konzeptionelle Arbeit in der praktischen Forschung. Der von dem Altphilologen Martin Mueller geprägte Begriff des »Scalable Reading $\ll^{2}$ stellt einen solchen konzeptionellen Versuch dar. Er ist forschungspoli-

\footnotetext{
1 Vgl. den Konferenzbericht von Eggert, Cornelius: »Scalable Reading. Paul Heyses Deutscher Novellenschatz zwischen Einzeltext und Makroanalyse«. In: Zeitschrift für Germanistik 1 (2016).

2 Vgl. https://scalablereading.northwestern.edu/ .
}

T. Weitin $(\triangle)$

Technische Universität Darmstadt, Darmstadt, Deutschland

E-Mail: weitin@linglit.tu-darmstadt.de 
tisch attraktiv, weil er Ansätze denkbar werden lässt, die die polemische Frontstellung von close und distant reading überwinden. ${ }^{3}$ Er birgt dabei zugleich die Gefahr, sich die mixed methods-Ansätze, nach denen so viele suchen, zu einfach vorzustellen. Die Methodenmetapher vom skalierbaren Lesen impliziert die Vorstellung eines stufenlosen Hin-und-her-Schaltens oder Zoomens zwischen der durch Lektüre erschließbaren Ebene und abstrakten Repräsentationen von Text, die die Grundlage quantitativer Analysen bilden. So irreführend diese Vorstellung ist, so deutlich zeigen gerade diejenigen Arbeiten, die bestimmten quantitativen Verfahren zur literaturwissenschaftlichen Korpusanalyse zum Durchbruch verholfen haben, dass die AutorInnen ihr Korpus jeweils sehr gut kannten. ${ }^{4}$ Unser Ansatz bei der Arbeitstagung zum Scalable Reading des Deutschen Novellenschatzes setzte darauf, dass die Verbindlichkeit des gemeinsamen Gegenstandes uns helfen würde, genauer zu erkennen, worin die methodischen Schwierigkeiten bestehen, die alle Versuche, Mikround Makroanalyse zusammenzuführen, unweigerlich begleiten.

In der zweiten Hälfte des 19. Jahrhunderts gilt die Novelle als Inbegriff von Massenliteratur, und es ist dieses Verständnis, das Heyse und Kurz bei ihrer Sammlung immer wieder beschäftigt. Das Titelblatt (s. Abb. 1) ziert einerseits eine mit gepflegter Lektüre beschäftigte Dame, andererseits fallen aus einem Füllhorn ungeordnet Büchermassen auf einen größer werdenden Haufen. Das Bild ist ein guter Ausdruck für die zwiespältige Haltung, die die Herausgeber gegenüber dem literarischen Massenmarkt ihrer Zeit einnehmen. Sie verstehen ihre Sammlung als den Versuch, aus der »wachsende[n] Menge der Tages- und Wochenblätter« dasjenige auszuwählen, was des »Aufhebens werth « ist, und sind sich doch im Klaren darüber, dass sich dem »Zeitgeschmack « gegenüber »ein doctrinärer Eigensinn « verbietet. ${ }^{5}$ Dem normativen Bemühen um Kanonisierung tritt die Einsicht an die Seite, dass sich Autoren wie Herausgeber mit der Masse werden arrangieren müssen. Die populäre Gattung der Novelle war nicht nur der Inbegriff von Massenproduktion (Heyse allein schrieb insgesamt 177), sondern auch des immer Gleichen oder jedenfalls massenhaft Ähnlichen, das daraus entstand. Mit seiner oft belächelten Falkentheorie gab Heyse in der Einleitung zum Novellenschatz konkrete Hinweise, wie unter diesen Produktionsbedingungen überhaupt noch eine Distinktion möglich war. Schriftstellerinnen und Schriftsteller sollten ihren Stoff auf das Vorhandensein eines zentralen Grundmotivs prüfen, anhand dessen sich der $»$ Inhalt in wenige Zeilen $\ll^{6}$ zusammenfassen lässt. Heyse verlangt von diesem Grundmotiv eine »starke Silhouette «, was auch auf die mentale Repräsentation beim Leser hindeutet, die hier offensichtlich mitgedacht wird. Schließlich soll die »einfache Form« auf »das Spezifische« getestet werden, »das diese Geschichte von tausend anderen unterscheidet «. ${ }^{7}$ Dabei entwickelt sich im Bewusstsein der notwendigen Distinktion auf dem literarischen Massenmarkt

\footnotetext{
3 Vgl. Weitin, Thomas: »Digitale Literaturwissenschaft«. In: DVJs 89.4 (2015), S. 651-656.

4 Vgl. dazu den Beitrag von Katharina Herget und Thomas Weitin in diesem Heft.

5 Heyse, Paul/ Kurz, Hermann: »Einleitung«. In: Dies. (Hg.): Deutscher Novellenschatz. München 1871, S. 10 (erstes Zitat), 22 (zweites Zitat), 14 (drittes und viertes Zitat).

6 Ebd., S. 19.

7 Ebd., S. 20.
} 
Abb. 1: Titelblatt des Deutschen Novellenschatzes

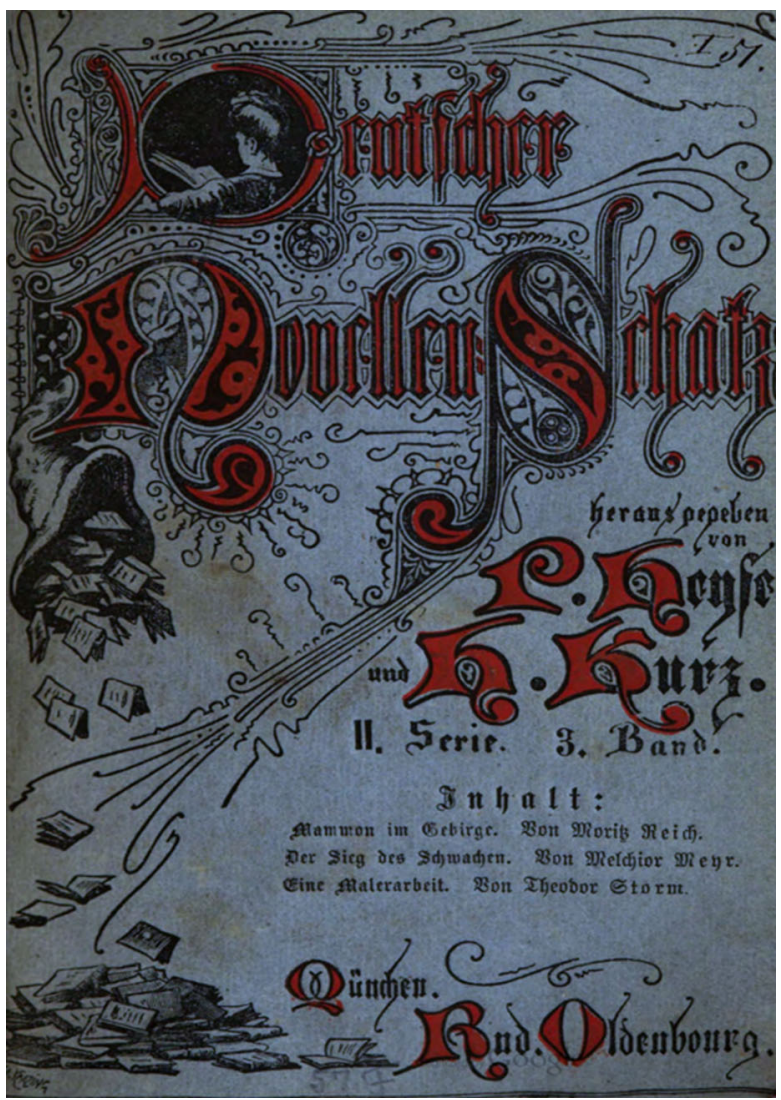

eine zeitgemäße Vorstellung von Originalität, die sich differentiell als Distanzmaß des einzelnen Textes zum Gesamtkorpus der Gattung bestimmt.

Die handreichungsartige Novellentheorie steht in systematischem Zusammenhang mit den Prinzipien der Textauswahl für den Novellenschatz. So, wie die Schriftstellerinnen und Schriftsteller angehalten werden, ihren jeweiligen Text schon in dem Bewusstsein sehr vieler ähnlicher Texte zu verfassen, sehen sich die Herausgeber auf der Suche nach geeigneten Novellen mit Textmassen konfrontiert. Die ursprüngliche Absicht einer chronologischen Literaturgeschichte der deutschsprachigen Novellistik, mit der sie an ihre auf 24 Bände angelegte Sammlung herangehen, wird der $»$ Unabsehbarkeit des Stoffes $\aleph^{8}$ wegen aufgegeben. Stattdessen versuchen die Herausgeber, innerhalb einzelner Bände Formreihen nach dem Kriterium des >Tons $<$ zu bilden, um dadurch etwa den Unterschied zwischen romantischem und realistischem Stil deutlich werden zu lassen. Wenn es am Ende der Einleitung heißt, der Novellenschatz solle eine »Mustersammlung « darstellen »für eine künftige Geschichte der Novelle $\ll,{ }^{9}$ erinnern wir uns, wie diese Literaturgeschichte im Übergang zur Mo-

\footnotetext{
8 Ebd., S. 22.
}

9 Ebd., S. 24. 
derne geschrieben worden ist und heute auf der Basis digitaler Methoden erneut geschrieben wird: als Geschichte der Form. ${ }^{10}$

Die Herausgeber des Novellenschatzes verfügen über ein ausgeprägtes realistisches Epochenbewusstsein, das ihre Vorstellung von der Gattung und ihre Textauswahl bestimmt. 49 der 86 Novellen stammen aus der Zeit nach 1848, die beiden ältesten von 1811, die jüngste von 1875. Versammelt sind fast ausschließlich Einzeltexte, nur vier Autoren sind mit zwei Texten vertreten. 12 weiblichen stehen 70 männliche Autoren gegenüber.

Wir eröffnen dieses Heft mit dem Beitrag von Fotis Jannidis, der der marktstrategisch kalkulierten Novellentheorie unserer Sammlung mit verschiedenen quantitativen Verfahren nachgegangen ist, um zu untersuchen, ob und inwieweit sich die Vorstellungen, die die Herausgeber von ihrer Auswahl propagieren, korpusanalytisch erhärten lassen. Je nach Operationalisierung fallen die Ergebnisse unterschiedlich aus. Eine stilometrische Analyse der Distanzmaße zwischen allen 86 Novellen zeigt Eichendorffs Die Glücksritter als einen derjenigen Vor-48er-Texte, der besonders stark als stilistisches Vorbild zu erkennen ist. Die Herausgeber versuchen dagegen, diese Ähnlichkeit zu relativieren, und betonen die klare Absetzung der Realisten von Eichendorff. ${ }^{11}$ Als »Stilmuster ${ }^{12}$ hatten sie vor allem Goethe im Sinn. Heyses Forderung nach einer klaren Silhouette der Novelle lässt sich im Sinne eines stark reduzierten Personals mit wenigen zentralen Figuren verstehen und entsprechend quantitativ erfassen. Allerdings erbringt diese Operationalisierung im Vergleich mit einem Romankorpus keinen sichtbaren Unterschied, was sich interessanterweise ändert, sobald man nach der Interaktionsstruktur der Figuren fragt. Ein weiterer Vergleich zeigt, dass die spezielle Auswahl von Heyse und Kurz die versammelten Texte von Frauen stärker in eine Position des Unterschiedenseins bringt als das Vergleichskorpus aus Romanen.

Der Beitrag von Thomas Weitin und Katharina Herget diskutiert am Topic Modeling des Novellenschatzes, welche Schwierigkeiten die quantitative Analyse semantischer Strukturen in literarischen Texten mit sich bringt. Über das Topic Modeling werden Wörter herausgefiltert, deren gemeinsames Auftreten wahrscheinlich ist. Die ermittelten semantischen Muster lassen sich Themen zuordnen, die unseren Lektürebefunden und dem Wissen über Gattung und Epoche entsprechen. Wer sein Korpus gut kennt, kann darüber hinaus weitere Beobachtungen machen. Weitin/Herget ge-

10 Vgl. dazu ausführlich: Weitin, Thomas: »Selektion und Distinktion. Pauls Heyses und Hermann Kurz' Deutscher Novellenschatz als Archiv, Literaturgeschichte und Korpus«. In: Daniela Gretz/Nicolas Pethes (Hg.): Archiv/Fiktionen. Verfahren des Archivierens in Literatur und Kultur des langen 19. Jahrhunderts. Freiburg 2016, S. 385-408.

11 Auf Eichendorff, den die Herausgeber »den letzten Romantiker« nennen, folgt im dritten Band Adolf Widmanns Die katholische Mühle, die die klare Absetzung des Realismus exemplarisch vorführen soll. Diese Novelle zeige zwar »in Stoff und Tonart« eine Verwandtschaft mit dem »Grundton der Eichendorff'schen Dichtung«. »Und doch bedarf es nur eines Blickes in unsere Novelle, um nicht nur jeden Gedanken der Nachahmung zu verscheuchen, sondern auch inne zu werden, daß hier das Stoff- und Stimmungsgebiet der Romantik mit vollster Klarheit für den modernen Realismus erobert worden ist. « (Heyse/ Kurz: Deutscher Novellenschatz. Bd. 3. Einleitung zu Adolf Widmann, S. 163f.).

12 Paul Heyse an Hermann Kurz am 6. Juni 1870, zitiert nach Walkhoff, Monika: Der Briefwechsel zwischen Paul Heyse und Hermann Kurz in den Jahren 1869-1873 aus Anlass der Herausgabe des »Deutschen Novellenschatzes«. Dissertationstyposkript. München 1967, S. 37. 
hen dem Befund nach, dass in einer nicht geringen Zahl von Fällen alle TopicWörter aus ein und demselben Text stammen, dessen Inhalt sich darüber gut zusammenfassen lässt. Das muss irritieren, weil die Texte im Preprocessing in kleine Einheiten zerlegt worden waren. Die in Anlehnung an Heyse so genannten $>$ Falkentopics< werden beim Topic Modeling ihrer geringeren semantischen Kohärenz wegen meist nur als Verzerrung des Ergebnisses wahrgenommen. Hier wird dagegen ihr >topischer Charakter diskutiert: Namen, Eigennamen und Hinweise auf Orte geben Anhaltspunkte für das Setting der betreffenden Novellen.

Katja Mellmann untersucht am Novellenschatz, wie sich anhand von Zeit- und Ortsangaben sprachliche Signale für den >epischen Modus< der Novellen isolieren und messen lassen. Darunter wird in Anlehnung an Käte Hamburger ein spezifischer Wechsel in einen konjunktivischen Modus verstanden, der sich von der indikativischen Wirklichkeitsaussage absetzt. Evolutionsbiologisch betrachtet dient der Epitiv dazu, die wachsende Menge von Informationen durch eine eigene Bereichssyntax für >entpflichtete Redeく zu verwalten. Dieser angeborene kognitive Modus bedarf der sprachlichen Aktivierung, wobei sich Mellmann im vorliegenden Beitrag auf zeitliche und räumliche Signale der Unbestimmtheit konzentriert, die in einer Analyse aller 86 Erzähleingänge ermittelt werden. Wie erwartet kristallisiert sich dabei das heterodiegetische Erzählen als der bevorzugte Träger für den epischen Modus heraus, wobei ein Erzählen mit historischer Ortsmarkierung, aber unbestimmter Zeitangabe dominant ist. Epizität erscheint damit nicht unbedingt als gattungsdefinierendes Merkmal, wohl aber als eine Eigenschaft, die für die Novelle im Zeichen des Realismus als typisch gelten kann.

Bent Geberts Beitrag betrachtet den Novellenschatz von Heyse und Kurz vor dem Hintergrund konkurrierender Sammlungsprojekte im 19. Jahrhundert, die die Herausgeber weitgehend ausblenden oder gar aktiv verdrängen. Das gilt vor allem für die novellistischen Kurzerzählungen des Mittelalters, die in zeitgenössischen Editionen, Sammlungen und Kommentaren überaus präsent waren. Vor allem Hermann Kurz dürfte sie als Tübinger Bibliothekar gut gekannt haben, Heyse behauptet dagegen in der Einleitung zum Novellenschatz in einer bemerkenswert kontrafaktischen Vorgeschichte ihren Untergang, um seine eigene Sammlung als ein Archiv der Gattung zu profilieren, das dann mit Goethe beginnen kann. Den Heyseschen Falkentest, so argumentiert Gebert dagegen in seinem Beitrag, würden viele der mittelalterlichen Mären bestehen.

Eine Basis, um die Heysesche Literaturpolitik korpusanalytisch zu hinterfragen, stellt die langjährige Arbeit von Friedrich Michael Dimpel dar, der ein großes Korpus von Mären und Novellen aufbaut, zu dem seit unserer Tagung auch eine Auswahl aus dem Novellenschatz gehört. Im vorliegenden Beitrag erläutert Dimpel am Beispiel von Victor von Scheffels Hugideo das narratologisch orientierte Annotationssystem, das er mit seinem Korpus entwickelt. Anhand seiner Tagsets und der Annotationsrichtlinien führt Dimpel vor, wie die hermeneutisch geprägten Modelle zur Textauszeichnung immer wieder durch die Ambiguität ihres Gegenstandes herausgefordert werden. Wir erhalten damit einen wichtigen Einblick in den Maschinenraum des Scalable Reading.

Auch Nicolas Pethes interessiert sich in seinem Beitrag für das Verhältnis des Deutschen Novellenschatzes zu anderen zeitgenössischen Sammlungen und zum Dis- 
positiv des Sammelns von Texten unter den Bedingungen von Massenproduktion. Dabei steht die Verbindung zum Fall (Kasus) und zur Fallgeschichte im Mittelpunkt, die der Novelle als einfacher Form gattungspoetisch eigen ist. Pethes untersucht in Stichproben die Fallsemantik und zeigt, wie einzelne Texte der Sammlung den Umgang mit großen Textmengen thematisieren.

In ihrem sprachwissenschaftlichen Beitrag untersucht Christin Schätzle am Novellenschatz-Korpus den Einsatz des Genitivs als Stilmittel. Im Vergleich mit Texten aus dem 20. und 21. Jahrhundert zeigt sich das Korpus einerseits innerhalb der bekannten Tendenz zum Genitivschwund. Andererseits sind nicht alle Genitivkonstruktionen von diesem Schwund betroffen. Wo er verbleibt, wird der Genitiv vor allem als Stilmittel verwendet. Vergleiche zwischen einzelnen Bänden innerhalb des Novellenschatzes zeigen große Unterschiede und lassen z.B. erkennbar werden, dass die stärker dialektal und an der Volkssprache orientierten Dorfgeschichten eine besonders niedrige Genitivfrequenz aufweisen.

Die Arbeitstagung »Scalable Reading « war ein methodisches Experiment, von dem keine fertigen Ergebnisse erwartet werden durften. Was die Beiträge des vorliegenden Heftes eint, ist nicht zuletzt ihr Charakter als work in progress. Das methodische Fachgespräch wird fortzusetzen sein, auch als Teil eines größeren korpusanalytischen Projekts zum Thema Mixed Methods, das vom Deutschen Novellenschatz seinen Ausgang nimmt. ${ }^{13}$

${ }^{13}$ Die VolkswagenStiftung fördert das Projekt »Reading at Scale. Mixing Methods in Literary Corpus Analysis ab 2017 im Programm »Mixed Methods in the Humanities«. 